\title{
Fibre-based imaging: new challenges
}

\author{
Martin Plöschner $^{a}$, Branislav Straka $^{c}$, Kishan Dholakia $^{d}$ and Tomáš Čižmár ${ }^{a, b}$ \\ ${ }^{a}$ School of Engineering, Physics \& Mathematics, University of Dundee, Dundee, DD1 4HN, \\ Scotland \\ ${ }^{b}$ School of Medicine, University of St. Andrews, North Haugh, St. Andrews KY16 9TF, UK; \\ ${ }^{c}$ Faculty of mechanical engineering, Brno university of Technology, Technicka 2896/2, 61669 \\ Brno, Czech Republic \\ ${ }^{d}$ SUPA, School of Physics and Astronomy, University of St. Andrews, North Haugh, St. \\ Andrews KY16 9SS, UK
}

\begin{abstract}
A novel minimally invasive micro-endoscopes utilizing disordered light within a standard multimode optical fibre have been introduced recently. The two most important limitations of this exciting technology are (i) the lack of bending flexibility (transformation matrix is only valid as long as the fibre remains stationary) and (ii) high demands on computational power, making the performance of such systems slow. Here we discuss possible routes to address the later one: We introduce a GPU toolbox to make this powerful technique faster and more accessible to bio-medical researchers.
\end{abstract}

Keywords: Digital holography, multimode waveguides, fluorescence, microscopy

\section{INTRODUCTION}

An outstanding goal for photonics applied to biological systems is to obtain deeper penetration and indeed access without causing significant collateral photodamage or mechanical damage. Reducing the footprint of fibre based devices addresses this goal through miniaturisation which is therefore of paramount importance, especially for in vivo applications. In this regard, there has been a recent step change in this field with the advance of the shaping of light through thin multimode fibres $(\mathrm{MMF})^{1-4}$ whilst retaining the information capacity of bulky single mode fibre bundles. There has been progress towards the miniaturisation of endoscopic, ${ }^{2,5-10}$ nanosurgery and micromanipulation ${ }^{1,11}$ tools utilising the MMF. The MMF is poised to become a powerful tool in biomedical applications because of its very small cross-section leading to minimal invasiveness. However, the applications of multimode fibre technology are hindered by complex propagation characteristics of light inside the fibre[Ref]. For example, the diffraction limited spot at the fibre input excites many propagating modes inside the fibre. When the excited modes arrive at the other end of the fibre - their phase, amplitude and polarisation appear randomised and we observe a speckle-like output pattern. Although seemingly random, the output is, in fact, a combined result of mode dispersion and geometry dependent mode coupling that can be fully described by a linear transformation. In essence, the knowledge of the transformation matrix of the fibre allows to couple such a field to the proximal end of the fibre that the desired pattern is generated at the distal end.

It was recently shown that the input-to-output mode transformation can be measured for a specific spatial configuration of the fibre ${ }^{1,2,5,11}$ and the result used to generate desired patterns at the distal end of the fibre, or vice-versa, for imaging the distal end pattern at the fibre input site. However, the previous implementations of the transformation matrix acquisition suffered from synchronisation issues between CCD and SLM which prevented to operate the hardware at the maximum possible speed. We have resolved the synchronisation issues in the toolbox by harnessing the computational power of modern GPUs that are able to deliver the mask on the SLM screen without substantial delay. This allows to acquire the transformation matrix at the maximum possible refresh rate of our SLM (Boulder Nonlinear Systems - 512x512). The speed gain is one of the important

Further author information: (Send correspondence to T.C.)

T.C.: E-mail: t.cizmar@dundee.ac.uk

Adaptive Optics and Wavefront Control for Biological Systems, edited by Thomas G. Bifano,

Joel Kubby, Sylvain Gigan, Proc. of SPIE Vol. 9335, 93350H · @ 2015 SPIE

CCC code: $1605-7422 / 15 / \$ 18 \cdot$ doi: $10.1117 / 12.2077693$

Proc. of SPIE Vol. $933593350 \mathrm{H}-1$ 
features of the toolbox as the full acquisition of the transformation matrix is a time consuming process that has to be repeated each time the fibre configuration is significantly changed. To the best of our knowledge, our toolbox offers the fastest implementation of transformation matrix acquisition using only the SLM during the transformation matrix measurements. Much faster acquisition times were achieved using the Digital Micromirror Device $^{12,13}$ or combination of two AODs and SLM, ${ }^{14}$ however, the methods measured just a fraction of the full transformation matrix, giving capability to display only a single point at the fibre output. Our toolbox measures the full transformation matrix which allows to display any pattern at the fibre output.

The generation of output patterns used e.g. in micromanipulation studies ${ }^{1,11}$ normally requires significant computational time. As a result, a sequence of pre-calculated masks has to be used to realise the manipulation which precludes convenient interactive engagement with the system and is far from real-time implementation. Superposition of holograms generating an array of output spots also suffers from undesired interference. In our toolbox, we address both of these issues. The full transformation matrix of the multimode fibre is stored in the GPU memory and, as a result, the holograms corresponding to the desired patterns can be computed on-demand in approximately $20 \mathrm{~ms}$ for 120 output points. Unfortunately, the interference effects of neighbouring spots, especially in closed packed patterns, decrease the quality of the output. This effect can only be partially removed using the iterative Gerchberg-Saxton algorithm. ${ }^{1}$ In our toolbox, we present a complete removal of the interference in the output patterns by implementing an acousto-optic deflector (AOD) into the setup. This allows to display the fibre output points in time-discrete manner, preventing the interference. The whole AOD cycle, for 120 points, is $0.25 \mathrm{~ms}$ long, much shorter then the time required for hologram calculation.

\section{PRINCIPLES OF TRANSFORMATION MATRIX MEASUREMENT}

The measurement of the transformation matrix is performed by scanning a diffraction limited spot across the input facet of the fibre. Each spot position, $(u, v)$, is addressed by a specific diffraction grating on the SLM. This method is different from our previous implementation, where the SLM was divided into discrete regions, each of which represented a plane-wave incident at some specific angle on a fibre input facet. This new implementation, first demonstrated in Ref., ${ }^{11}$ has several advantages. First, it utilises the whole area of the SLM during the calibration process, using the maximum laser power for each spot. Second, it provides a direct means of mode discretisation of the input facet of the fibre by assigning a specific grating to each mode on the orthogonal grid $(u, v)$. Finally, the optimised holograms are at a full resolution of the SLM by default, without the need to create very small discrete regions on the SLM.

Each spot position $(u, v)$, at the input facet, excites a particular linear superposition of propagating modes inside the MMF. The propagating modes subsequently create certain field pattern at the distal end of the fibre having coordinates $(x, y)$. The resulting pattern is then interfered with the collimated Gaussian output of the Single Mode Fibre (SMF). ${ }^{1}$ The simultaneous coupling of light into SMF and MMF is realised on the SLM by complex superposition of corresponding gratings and taking only the phase term of the resulting complex matrix. The phase of the spot in $(u, v)$ is subsequently altered $N$ times over a range of $2 \pi n$. The images $(120 \times 120$ pixels) acquired by the CCD (Basler piA640-210gm) for each of these phase steps are subsequently saved in the GPU memory. The resulting images can be used to extract the input $(u, v)$-mode phase $\Delta \phi_{u v}(x, y)$ and amplitude $A_{u v}(x, y)$ information for the output field in any point $(x, y)$. The process is repeated for all input spot coordinates $(u, v)$. The well defined spot in arbitrary $(x, y)$ can be generated by constructive interference of the output fields in $(x, y)$. To do that we have to adjust the phase and amplitude of the input modes by multiplying the gratings generating them by a complex number $A_{u v}(x, y) e^{-i \Delta \phi_{u v}(x, y)}$ and sum all such adjusted gratings in a complex manner. This process is intuitive but relatively slow. Equivalent approach is to FFT the complex matrix $A_{u v}(x, y) e^{-i \Delta \phi_{u v}(x, y)}$ which leads directly to the desired SLM hologram. The FFT process is fast enough to calculate a hologram focusing the spot into an arbitrary position $(x, y)$ in real-time (see Multimedia 1) from the raw acquired data. However, it is much faster to save the input modes amplitude and phase matrices $M_{u v}(x, y)=A_{u v}(x, y) e^{-i \Delta \phi_{u v}(x, y)}$ for all output points $(x, y)$ and calculate the holograms by applying FFT to $M_{u v}(x, y)$ matrix itself. The FFT step is unavoidable at current generation GPUs as the complete set of holograms for all output points exceeds the amount of memory for most top of the range cards.

The measurement of the transformation matrix for $25 \times 25$ input modes for $N=8$ and $n=2$, and both input polarisations, takes approximately $49 \mathrm{~s}$ when running at a maximum refresh rate of the SLM $(204 \mathrm{~Hz})$. The

Proc. of SPIE Vol. $933593350 \mathrm{H}-2$ 
above corresponds to 10000 frames that require the acquisition of the output fields to be carefully synchronised with the CCD. A routine is implemented into our software to check if the synchronisation of the CCD and the SLM is present. This routine is based on a rotating spot that allows for unambiguous matching of the CCD frames to the SLM holograms. This routine revealed a constant delay of two frames between the displaying of the hologram and the acquisition of the hologram-related frame in our system. This delay can probably be attributed to several software and hardware buffers in our system. However, as the delay is constant, its effect can easily be addressed within the software.

\section{AOD IMPLEMENTATION}

The holograms generating focused spots in arbitrary $(x, y)$ at the fibre output can be superposed in a complex way to generate arrays of spots at the fibre output. However, if the output spots are close to each other, they start to interfere with each other. These undesired interference effects can be partially removed by employing the G-S algorithm to the hologram. ${ }^{1}$ Although the G-S algorithm removes some of the undesired interference effects, it cannot remove the interference effects completely. As long as the output fields are generated and interfered coherently, interference effects will always negatively influence the output image. This undesirable effect can be removed by a method discussed in Ref. ${ }^{2}$ that constructs the output field point by point in time-discrete mode using the acousto-optic deflector (AOD) (Fig. 1). The hologram representing the desired output image

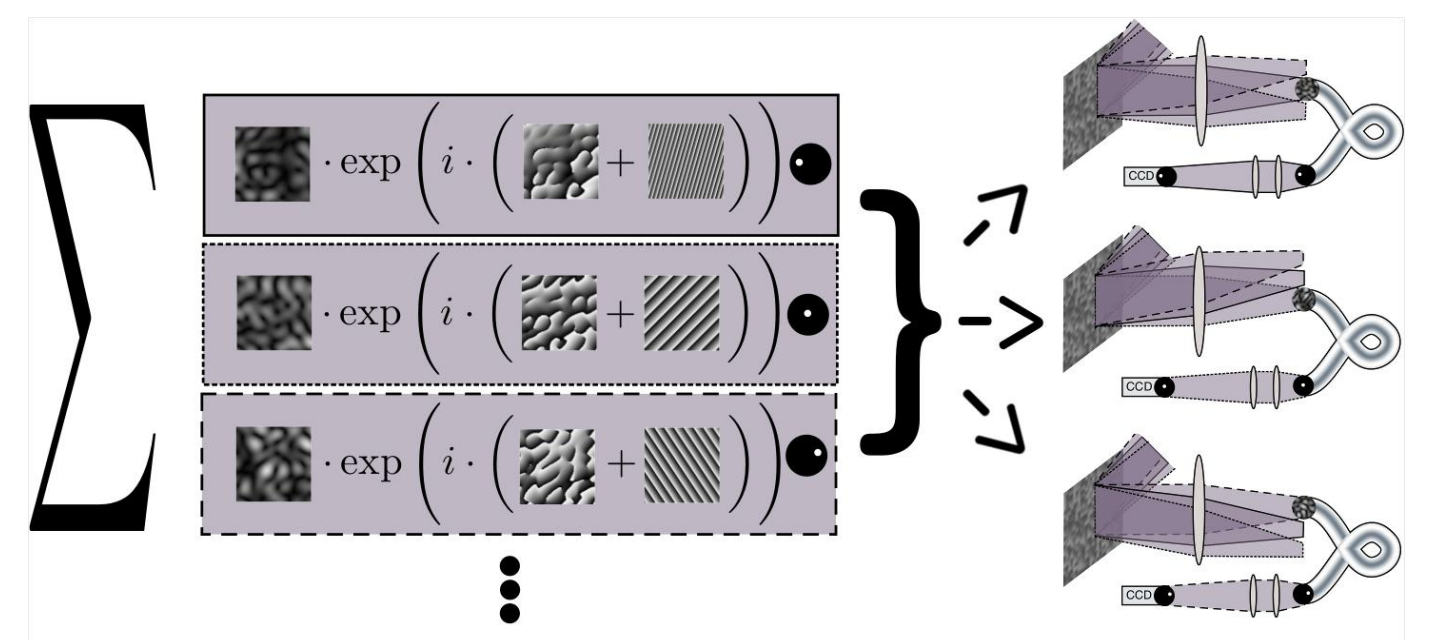

Figure 1. The beam deflected by AOD impinges on the SLM at an angle given by the current frequency of AOD. The hologram on the SLM consists of the complex superposition of masks focusing light into different spots $(x, y)$ at the fibre output. Each of the constituting masks has an added grating that cancels out one specific angle introduced by the AOD. Subsequently, only the input field corresponding to a mask with the right correction angle for the current AOD deflection will propagate through the fibre. In this way we can cycle between the spots at the output with a maximum refresh rate of the AOD without introducing any interference effects between the spots.

is created by a standard complex superposition of masks generating the focused spots in $(x, y)$. However, we carefully select the grating of the individual masks such that the effect of the angled incidence of the beam on the SLM, introduced by current AOD frequency, is exactly cancelled for one individual mask from the complete set. As a result, only the mask with correct grating for the current deflection of AOD is imaged on the fibre input facet - all other masks generate fields that miss the input facet for the current AOD deflection. As the AOD performs a full cycle scan through a defined set of frequencies, all the masks contained in the hologram get addressed, rendering the user defined image at the fibre output.

The complete hologram generation process, for 120 output points, can be done at a refresh rate of $50 \mathrm{~Hz}$ enabling interference free images to be generated on-the-fly at the output of the MMF for the first time to our knowledge. 


\section{REAL-TIME PATTERN GENERATION}

To demonstrate the real-time, on-the-fly generation of output images at the end of MMF, we supply a routine, within the GPU toolbox, that displays a rotating cube at the end of MMF (Fig. 2, Multimedia files). This
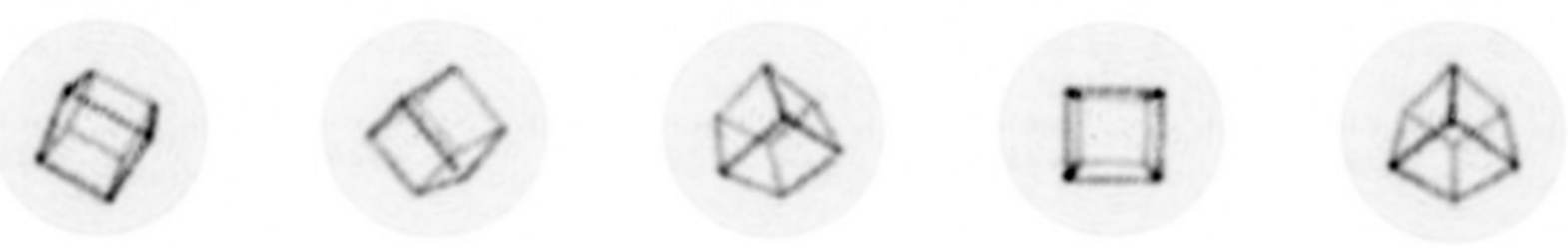

Figure 2. Projected 3D cube, created using 120 output points, generated at the distal end of the MMF with a refresh rate of $50 \mathrm{~Hz}$. The contrast ratio of the cube lines to the fibre background light is $20: 1$. Colours are inverted.

routine is completely interactive allowing the user to change the size of the cube and its rotational orientation at the refresh rate of $50 \mathrm{~Hz}$. To achieve this refresh rate we FFT the $M_{u v}(x, y)$ matrices for all desired output points $(x, y)$ with $128 \times 128$ resolution, scale to native SLM resolution of $512 \times 512$ and finally add the gratings. The full-resolution FFT at $512 \times 512$ and FFT at $256 \times 256$ allows refresh rate of $23 \mathrm{~Hz}$ and $42 \mathrm{~Hz}$ respectively, with essentially identical contrast (Fig. 3). Even the $64 \times 64$ FFT provides rotating cube with little change in contrast

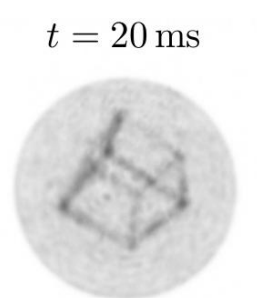

$F F T(32 \times 32)$

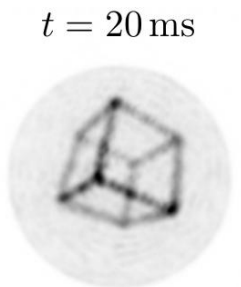

$\operatorname{FFT}(64 \times 64)$

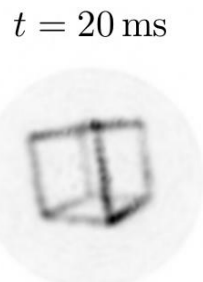

FFT $(128 \times 128)$

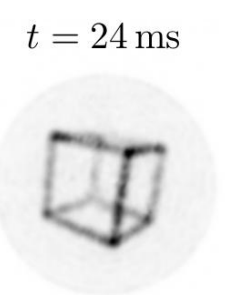

$\operatorname{FFT}(256 \times 256)$

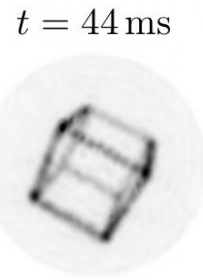

$\operatorname{FFT}(512 \times 512)$

Figure 3. The FFT resolution influences the contrast and computational time of the generated pattern. The contrast is significantly worse only for $32 \times 32$ and $64 \times 64$ FFT and the computational time is significantly longer only for $512 \times 512$ FFT case. Therefore, the optimal trade-off between speed and contrast is for $128 \times 128$ or $256 \times 256$ FFT. Colours are inverted.

compared to the $512 \times 512 \mathrm{FFT}$. The only significantly worse case is $32 \times 32 \mathrm{FFT}$ that suffers from a significant loss of contrast possibly due to fibre coupling of neigboring orders of the created grating. All the FFT resolution cases, up to $256 \times 256$ have roughly the same computational time of $20 \mathrm{~ms}$. The constant computational time can be explained by function overhead prohibiting lower computational times. The optimum trade-off between speed and quality is therefore at the FFT resolution of either $128 \times 128$ or $256 \times 256$. The routine can be easily supplied with any user-defined pattern with any number of output points supported by the fibre and AOD system. As the computational complexity appears to increase only after $256 \times 256$ FFT, the high refresh rate for larger number of points than presented here, can be maintained by decreasing the resolution of FFT.

\section{CONCLUSION}

We have designed an open-source GPU accelerated toolbox for shaping the light transmission through a MMF. The toolbox has several modalities. It allows fast and complete determination of transformation matrix of the MMF for both input polarisations. The results of the transformation matrix are stored in GPU memory and the data can be used to generate arbitrary patterns at the distal end of the fibre in real-time. The interference effects between neighbouring points at the output facet were addressed by implementation of AOD into the system. The AOD allows for an extremely fast, but crucially time-discrete display of the output spots, which completely eliminates the interference effects from the output pattern. The GPU routine driving the system with AOD in real-time, and generating holograms on-the-fly, was demonstrated on a fully interactive model of 
rotating cube being displayed at the distal end of the MMF. The routines in the GPU toolbox are not limited to MMFs. The transformation matrix measurement, and the output image formation can be utilised in any linear optical system suffering from aberrations. As such our GPU toolbox should prove useful for a wide community of researchers interested in exploiting complex linear media and particularly for those wishing to exploit the MMF in endoscopic applications.

\section{REFERENCES}

[1] Čižmár, T. and Dholakia, K., "Shaping the light transmission through a multimode optical fibre: complex transformation analysis and applications in biophotonics," Optics Express 19(20), 18871-18884 (2011).

[2] Čižmár, T. and Dholakia, K., "Exploiting multimode waveguides for pure fibre-based imaging," Nature Communications 3, 1027 (2012).

[3] Papadopoulos, I. N., Farahi, S., Moser, C., and Psaltis, D., "Focusing and scanning light through a multimode optical fiber using digital phase conjugation," Optics Express 20(10), 10583-10590 (2012).

[4] Di Leonardo, R. and Bianchi, S., "Hologram transmission through multi-mode optical fibers," Optics Express 19(1), 247-254 (2011).

[5] Choi, Y., Yoon, C., Kim, M., Yang, T. D., Fang-Yen, C., Dasari, R. R., Lee, K. J., and Choi, W., "Scannerfree and wide-field endoscopic imaging by using a single multimode optical fiber," Physical Review Letters 109(20), 203901 (2012).

[6] Papadopoulos, I. N., Farahi, S., Moser, C., and Psaltis, D., "Increasing the imaging capabilities of multimode fibers by exploiting the properties of highly scattering media," Opt. Lett. 38, 2776-2778 (Aug 2013).

[7] Thompson, A. J., Paterson, C., Neil, M. A., Dunsby, C., and French, P. M., "Adaptive phase compensation for ultracompact laser scanning endomicroscopy," Optics Letters 36(9), 1707-1709 (2011).

[8] Papadopoulos, I. N., Farahi, S., Moser, C., and Psaltis, D., "High-resolution, lensless endoscope based on digital scanning through a multimode optical fiber," Biomedical optics express 4(2), 260 (2013).

[9] Nasiri, R., Mahalati, Gu, R. Y., and Kahn, J. M., "Resolution limits for imaging through multi-mode fiber," Optics Express 21(2), 1656-1668 (2013).

[10] Choi, Y., Yoon, C., Kim, M., Yang, J., and Choi, W., "Disorder-mediated enhancement of fiber numerical aperture," Optics Letters 38(13), 2253-2255 (2013).

[11] Bianchi, S. and Di Leonardo, R., "A multi-mode fiber probe for holographic micromanipulation and microscopy," Lab on a Chip - Miniaturisation for Chemistry and Biology 12(3), 635-639 (2012).

[12] Caravaca-Aguirre, A. M., Niv, E., Conkey, D. B., and Piestun, R., "Real-time resilient focusing through a bending multimode fiber," Optics Express 21(10), 12881-12887 (2013).

[13] Conkey, D. B., Caravaca-Aguirre, A. M., and Piestun, R., "High-speed scattering medium characterization with application to focusing light through turbid media," Optics Express 20(2), 1733-1740 (2012).

[14] Cui, M., "A high speed wavefront determination method based on spatial frequency modulations for focusing light through random scattering media," Optics Express 19(4), 2989-2995 (2011). 